\title{
Modulation of Actinorhodin Biosynthesis in Streptomyces lividans by Glucose Repression of afsR2 Gene Transcription
}

\author{
EUNG-SOO KIM, ${ }^{1,2}$ HEE-JEON HONG ${ }^{3}$ CHA-YONG CHOI ${ }^{3}$ AND STANLEY N. COHEN ${ }^{2 *}$ \\ Department of Genetics, Stanford University School of Medicine, Stanford, California 94305-5120, ${ }^{1}$ and Department of \\ Environmental Science, College of Natural Science, Hankuk University of Foreign Studies, Kyungi-Do, ${ }^{2}$ and Division of \\ Chemical Engineering, College of Engineering, Seoul National University, Seoul, ${ }^{3}$ Korea
}

Received 16 October 2000/Accepted 8 January 2001

\begin{abstract}
While the biosynthetic gene cluster encoding the pigmented antibiotic actinorhodin (ACT) is present in the two closely related bacterial species, Streptomyces lividans and Streptomyces coelicolor, it normally is expressed only in $S$. coelicolor-generating the deep-blue colonies responsible for the $S$. coelicolor name. However, multiple copies of the two regulatory genes, afs $R$ and afsR2, activate ACT production in $S$. lividans, indicating that this streptomycete encodes a functional ACT biosynthetic pathway. Here we report that the occurrence of ACT biosynthesis in S. lividans is determined conditionally by the carbon source used for culture. We found that the growth of $S$. lividans on solid media containing glucose prevents ACT production in this species by repressing the synthesis of afsR 2 mRNA; a shift to glycerol as the sole carbon source dramatically relieved this repression, leading to extensive ACT synthesis and obliterating this phenotypic distinction between $S$. lividans and $S$. coelicolor. Transcription from the afsR 2 promoter during growth in glycerol was dependent on afs $R$ gene function and was developmentally regulated, occurring specifically at the time of aerial mycelium formation and coinciding temporally with the onset of ACT production. In liquid media, where morphological differentiation does not occur, ACT production in the absence of glucose increased as $S$. lividans cells entered stationary phase, but unlike ACT biosynthesis on solid media, occurred by a mechanism that did not require either afsR or afsR2. Our results identify parallel medium-dependent pathways that regulate ACT biosynthesis in $S$. lividans and further demonstrate that the production of this antibiotic in S. lividans grown on agar can be modulated by carbon source through the regulation of afsR 2 mRNA synthesis.
\end{abstract}

The two closely related bacterial species Streptomyces lividans and Streptomyces coelicolor historically have been distinguished phenotypically by the ability of the latter to produce large amounts of the pigmented antibiotic actinorhodin (ACT), thus generating the deep-blue colonies responsible for the $S$. coelicolor name. While a functional biosynthetic gene cluster encoding ACT is present also in the S. lividans chromosome, normally little or no ACT is made. However, introduction of multiple copies of either of two ACT-regulating genes, afsR or afs $R 2(7,12,13,22)$ can circumvent the limitation on ACT production in S. lividans, increasing ACT biosynthesis dramatically in this species, as well as in $S$. coelicolor (19, $22,25)$. AfsR $(13,22)$ becomes an active regulator of antibiotic synthesis after it is phosphorylated by the AfsK protein $(9,14)$, whose gene is located downstream of afs $R$ on the chromosome of $S$. coelicolor (18). afsR2 (25), which is known as afs $S$ in $S$. coelicolor (19) and is located immediately $3^{\prime}$ to afs $R$, encodes a 63-amino-acid protein of unknown function. Neither afs $R$ nor afsR2 is absolutely required for ACT biosynthesis, since production of this antibiotic is stimulated by overexpression of either gene in bacteria containing deletions in the other one (7, 25).

Here we report the surprising finding that the phenotypic limitation that historically has distinguished $S$. lividans cultures from those of $S$. coelicolor-namely, the inability of $S$. lividans

\footnotetext{
* Corresponding author. Mailing address: Stanford University School of Medicine, Department of Genetics, M-320, Stanford, CA 943055120. Phone: (650) 723-5315. Fax: (650) 725-1536. E-mail: sncohen @stanford.edu.
}

in its native state to produce significant amounts of ACT from the chromosomal biosynthetic gene cluster-can be remedied simply by changing the culture conditions. We show that ACT biosynthesis in S. lividans is repressed by glucose in the media commonly used for cell growth and that a change of carbon source can promote $S$. lividans ACT production by elevating afsR2 mRNA-increasing cellular pigmentation to a level characteristic of $S$. coelicolor and obliterating this phenotypic distinction between the two Streptomyces species.

\section{MATERIALS AND METHODS}

Bacterial strains and plasmids. S. lividans TK21, SL94, and SL41 were described previously (25), and a restriction map of the $a f_{S} R$ and $a f_{s} R 2$ gene region in S. lividans is shown in Fig. 1. S. lividans SL94-1 is a TK21 derivative containing an $a f s R 2:: t s r$ segment integrated into the chromosome by targeted gene disruption (described in the section on targeted mutagenesis). E. coli DH5 $\alpha$ was used as a host to generate DNA for plasmid pMOV96-1. Other organisms and plasmids used in this study were previously described $(11,21,25)$.

Media and culture conditions. Conditions for Streptomyces culture and liquid media (YEME) have been described previously (11). All Streptomyces strains were maintained on R5 agar media (11). For evaluation of ACT production, $S$. lividans pregerminated spores were streaked on minimal medium plates containing $0.5 \%$ glucose, $0.25 \%$ glycerol, or both as a carbon source, followed by 7 days of incubation at $30^{\circ} \mathrm{C}$. In liquid cultures, spores were added to a $250-\mathrm{ml}$ baffled flask containing $25 \mathrm{ml}$ of minimal medium that included either glucose or glycerol as the sole carbon source and were then shaken at $30^{\circ} \mathrm{C}$ for 3 days. Samples $(0.5 \mathrm{ml})$ were taken at various time points and examined for cell density, and the ACT concentrations (11) were determined. All experiments were done at least twice and found to generate consistent results. Escherichia coli cultures were grown on Luria-Bertani (LB) agar and liquid broth (21).

Transformation procedures. Competent $E$. coli cells and protoplasts of Streptomyces were prepared for DNA transformation as described previously $(11,21)$. Protoplasts of $S$. lividans were regenerated on R5 agar medium for $16 \mathrm{~h}$. Transformants were selected by overlaying the agar surface with $1 \mathrm{ml}$ of sterilized 


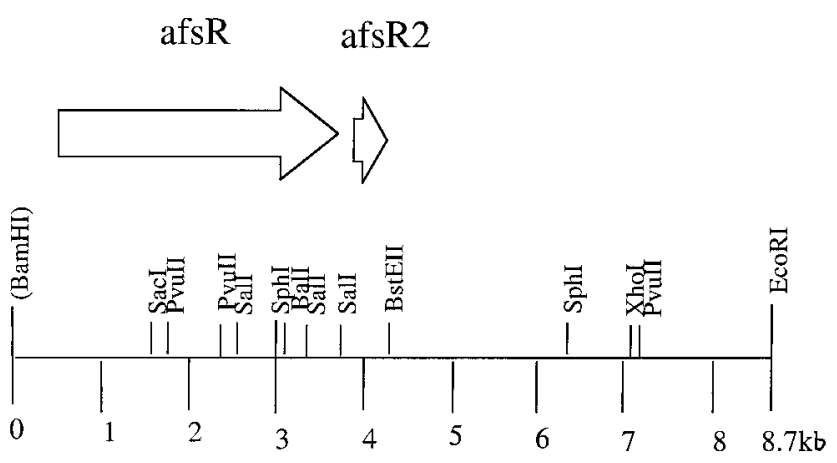

SL41

alkaline phosphatase (Promega), followed by ligation with a PCR-amplified ApaI fragment of $t s r$ using T4 DNA ligase (Promega). The pMOV96-1 is an afsR2containing pMOV96 derivative, of which afsR2 is interrupted at the unique ApaI site by the $t s r$ gene (see Fig. 3). pMOV96-1 was used to transform protoplasts of $S$. lividans TK21 for disruption of the chromosomal afsR2 gene. Thiostreptonresistant transformants, which were presumed to be single crossovers, arose at a frequency of $10^{-3}$ to $10^{-4}$ as determined by analysis of spores developed after 7 days. After several rounds of growth of single crossover transformants on selective media, segregants lacking vector sequences but containing an afsR 2 gene interrupted by a $t s r$ insertion (double crossovers) were isolated. One of these was designated SL94-1.

Southern blotting. For Southern hybridization, equal amounts of DNA samples were fractionated by electrophoresis in a $0.7 \%$ agarose gel and transferred to a positively charged nylon membrane (Amersham) as previously described (8). The DNA concentration was estimated from UV absorbance using the 260/280 $\mathrm{nm}$ ratio. Hybridization was carried out in $5 \times \mathrm{SSC}(1 \times \mathrm{SSC}$ is $0.15 \mathrm{M} \mathrm{NaCl}$ plus $0.015 \mathrm{M}$ sodium citrate) $-0.1 \% \mathrm{~N}$-lauroylsarcosine- $0.02 \%$ sodium dodecyl sulfate (SDS) $-1 \%$ blocking reagent at $58^{\circ} \mathrm{C}$ for $16 \mathrm{~h}$. The membrane was washed at high stringency $\left(2 \times\right.$ and $0.1 \times \mathrm{SSC}$ with $0.1 \%$ SDS at $\left.68^{\circ} \mathrm{C}\right)$. The DNA probe was labeled with a nonradioactive digoxigenin-labeled dUTP (Boehringer Mannheim).

S1 nuclease protection analysis and Northern blotting. The isolation of total RNA from Streptomyces and Northern blot hybridization were described in detail elsewhere (11). RNA was isolated from S. lividans plate cultures at the following three separate phases of growth on minimal agar plates using either glycerol or glucose as the sole carbon source: substrate mycelium, aerial mycelium, and spores. Cells were scraped from cellophane disks (8). The RNA concentration was normalized based on UV absorbance at $260 \mathrm{~nm}$ and verified by determining the amount of 5S RNA. The ${ }^{32}$ P-labeled DNA fragment used as a Northern blot hybridization probe was the same as that used for S1 mapping and was randomly labeled using a hexanucleotide priming kit (Amersham). The probe used to map the $a f s R 2$ promoter was generated using PCR from $S$. lividans TK21 total DNA as template using a $5^{\prime}$-end-labeled oligonucleotide primer internal to the afsR2 gene (afsR2II; 5'-TCCATCGTGGTGATCGCTTCGTT-3') and an unlabeled oligonucleotide (afsR2I; 5' -TCGACCGGCGGTGGCCGGGAGCGTT-3'). AfsR2II was labeled using $\left[\gamma^{-32} \mathrm{P}\right] \mathrm{ATP}\left(3,000 \mathrm{Ci} \mathrm{mmol}^{-1}\right.$; DuPont-NEN) (14). For this assay, $40 \mu \mathrm{g}$ of RNA and $25 \mathrm{fmol}$ of the probe were resuspended in 20 $\mu \mathrm{l}$ of sodium-trichloroacetic acid butter, hybridized at $45^{\circ} \mathrm{C}$ overnight following initial denaturation at $65^{\circ} \mathrm{C}$ for $15 \mathrm{~min}$, and digested with $\mathrm{S} 1$ nuclease. RNAprotected fragments were resolved on a $6 \%$ polyacrylamide sequencing gel.

\section{RESULTS}

Carbon-source-regulated afsR2-dependent stimulation of ACT production in $S$. lividans. Since afsR2 (afsS) overproduction from multiple gene copies borne by plasmids can induce $S$. lividans to synthesize large amounts of ACT, we sought to identify conditions that upregulate a single chromosomal copy

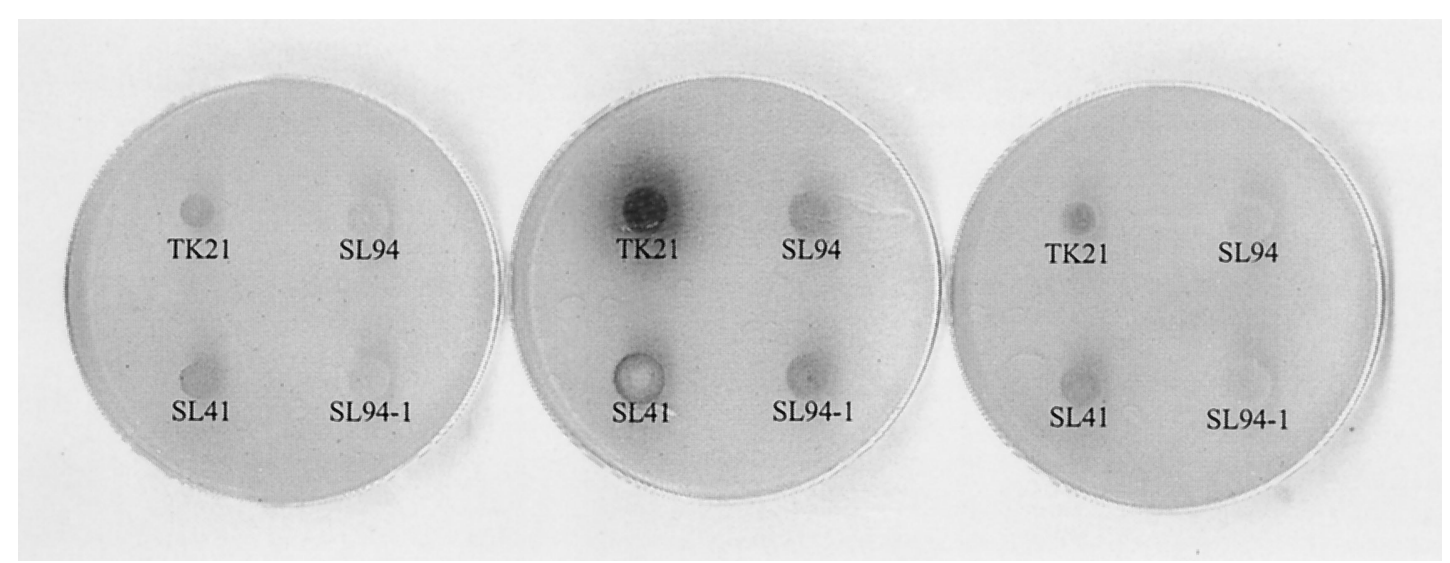

FIG. 2. ACT production by S. lividans (TK21) in the presence of glycerol. Portions (10 $\mu \mathrm{l})$ of diluted spore stocks of $S$. lividans TK21, SL41, SL94, and SL94-1 were spotted onto minimal-agar plates containing $0.5 \%$ glucose (left), $0.25 \%$ glycerol (middle), or both (right) as a carbon source. After a week of incubation at $30^{\circ} \mathrm{C}$, each plate was placed upside down for ammonia fuming as well as for photography. 
of $a f_{S R} 2$ using visually determined elevation of ACT biosynthesis as an assay. During these experiments we observed that ACT production by $S$. lividans TK21 $\left(a f s R 2^{+}\right)$, which normally is not observed during growth on minimal agar, even after an extended incubation period, when glucose is the carbon source (Fig. 2, left), occurred in large amounts when glycerol was substituted as the sole carbon source (Fig. 2, middle). The ability of TK21 to produce ACT in glycerol-containing media was reversed when glucose was also present (Fig. 2, right), indicating that ACT synthesis in the strain is subject to glucose repression. The TK21-derived S. lividans strain SL94, which lacks DNA segment of ca. $3.5 \mathrm{~kb}$ that includes both the afs 2 gene and the sequences adjacent to it (Fig. 1) (25), showed only limited ACT biosynthesis in all of these media (Fig. 2), suggesting that the ACT production observed during growth in glycerol requires genetic information absent in this strain.

The deletion in SL94 includes a small C-terminal segment of $a f_{s} R$ and all of $a f_{s} R 2$ (Fig. 1). Since the C-terminal segment is not required for $a f s R$ activity (13), we tentatively attributed the inability of SL94 to effectively synthesize ACT during growth in glycerol to the deletion of $a f_{s} R 2$. To confirm this presumption, we used targeted gene disruption to specifically inactivate the chromosomal afsR2 locus in TK21; this was done using a suicide vector, plasmid pMOV96-1 (25), in which the cloned afs 22 gene is interrupted by the thiostrepton resistance gene $(t s r)$. Following selection of thiostrepton-resistant transformants, a randomly selected clone (SL94-1) was analyzed by PCR and Southern blotting to confirm the absence of the vector and also the insertion of $t s r$ specifically in the chromosomal afsR2 gene (Fig. 3). On minimal-medium plates containing glycerol as the sole carbon source, SL94-1 showed results identical to those observed for SL94 (Fig. 2, middle), indicating that stimulation of ACT synthesis in S. lividans upon relief from glucose repression specifically requires $a f_{s} R 2$.

Expression of afsR2 is affected by carbon source, morphological differentiation, and the afs $R$ gene. As already noted, introduction of multiple plasmid-borne copies of the afsR2 gene can stimulate ACT biosynthesis in S. lividans (25). Our discovery that $S$. lividans TK21, which lacks multiple copies of afsR2, can produce large amounts of ACT when cultured on media containing glycerol but lacking glucose (Fig. 2), together with the finding that such stimulation of ACT production is dependent on afs $R 2$, suggested that carbon source regulation of ACT biosynthesis may be mediated through the control of afsR2 expression. This notion was tested directly by Northern blot analysis that compared steady-state levels of afsR 2 mRNA in TK21 and SL94 cells grown on minimal medium plates containing either glucose or glycerol as the sole carbon source. As seen in Fig. 4, afs $R 2$ transcripts of $\sim 400$ nucleotides nt were observed in TK21 when grown in glycerol but not when grown in glucose and were not detected in total RNA isolated from the SL94 mutant strain. Additionally, a high steady-state level of afsR 2 mRNA was seen only at the time of onset of both aerial mycelium formation and ACT production (day 4 of the cycle), indicating the developmental regulation of carbon source-mediated afs 2 transcription. S1 protection analysis (Fig. 4B) using an afsR2-specific probe identified the site of initiation of the carbon source-regulated transcripts, confirming that the transcripts shown by Northern blotting to be

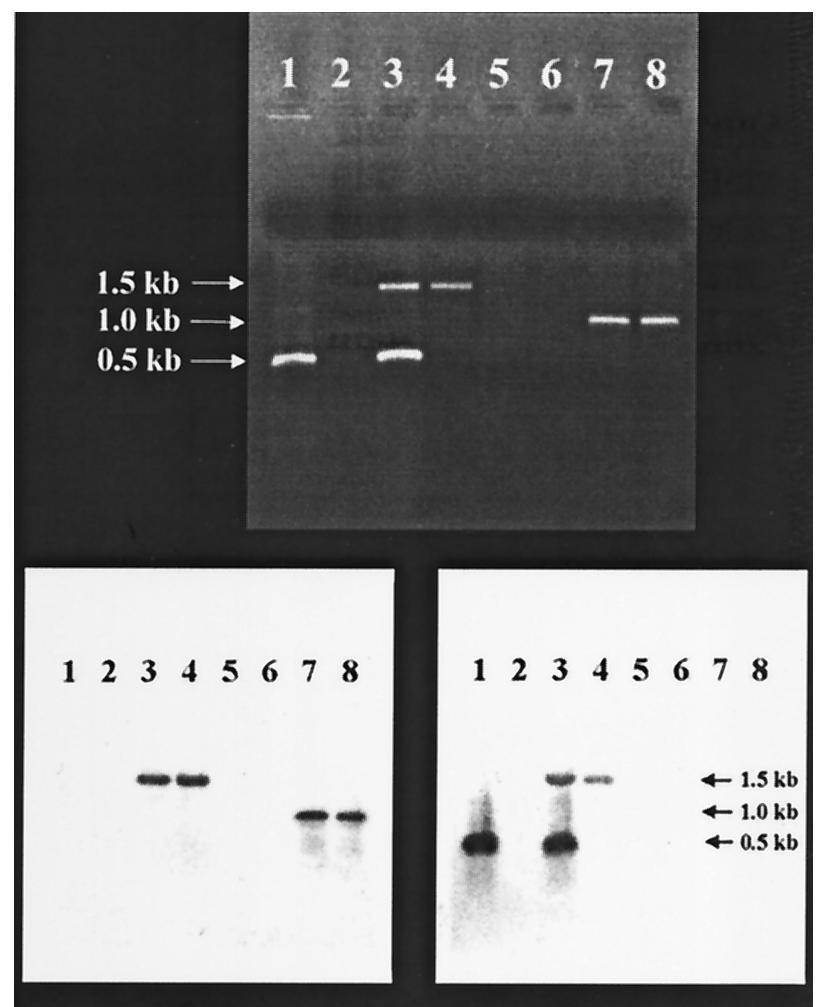

FIG. 3. (Top) Agarose gel electrophoresis of the PCR analysis with afsR2 (lanes 1 to 4 ) and tsr (lanes 5 to 8 ) primers. Each lane depicts a PCR result using as template the indicated chromosomal DNA. Lanes 1 and 5, TK21 chromosomal DNA; lanes 2 and 6, SL94 chromosomal DNA; lanes 3 and 7, single-crossover TK21 chromosomal DNA; lanes 4 and 8, double-crossover TK21 (SL94-1) chromosomal DNA. The single band shown in lane 1 is the afsR2 ( 500-bp) PCR fragment corresponding to the chromosomal copy of afsR2 in TK21. The absence of amplification products in lanes 2 and 5 reflects the absence afsR 2 and $t s r$ in the chromosome of SL94. Lane 3 shows two bands: the large one is the PCR product corresponding the $t s r$-interrupted afsR2 (ca. $1.5 \mathrm{~kb}$ ), and the small one is the intact afsR2. The one band seen in lane 4 is $t s r$-interrupted $a f s R 2$ resulting from the double crossover. Both lanes 7 and 8 show PCR products of $t s r$ secondary to the insertion of $t s r$ in the chromosomal copy of $a f s R 2$ in TK21. (Bottom) Southern blot hybridization analysis of PCR products using tsr (left) or afsR2 (right) probe DNA. The band sizes are indicated by the arrows. Lanes are as indicated in the top panel.

present at the onset of aerial mycelium formation are initiated at the afsR2 promoter.

During these studies we observed that ACT biosynthesis also did not occur in the $S$. lividans afsR deletion mutant, SL41, even when grown in glycerol in the absence of glucose (Fig. 2 and 4). However, since $S$. lividans cells containing multiple copies of $a f_{s} R 2$ but mutated in $a f_{s} R$ can produce large amounts of ACT (25), ACT biosynthesis cannot have an absolute requirement for $a f_{s} R$. Taken together with the findings described above, the absence of $a f s R 2$ transcripts in strain SL41 (Fig. 4) suggests that afsR, while not needed for ACT production per se in $S$. lividans, stimulates the transcription that occurs under normal conditions from the chromosomal afs 2 locus during growth on media containing glycerol.

ACT production in liquid cultures is independent of the AfsR2/AfsR pathway. While streptomycetes differentiate both 

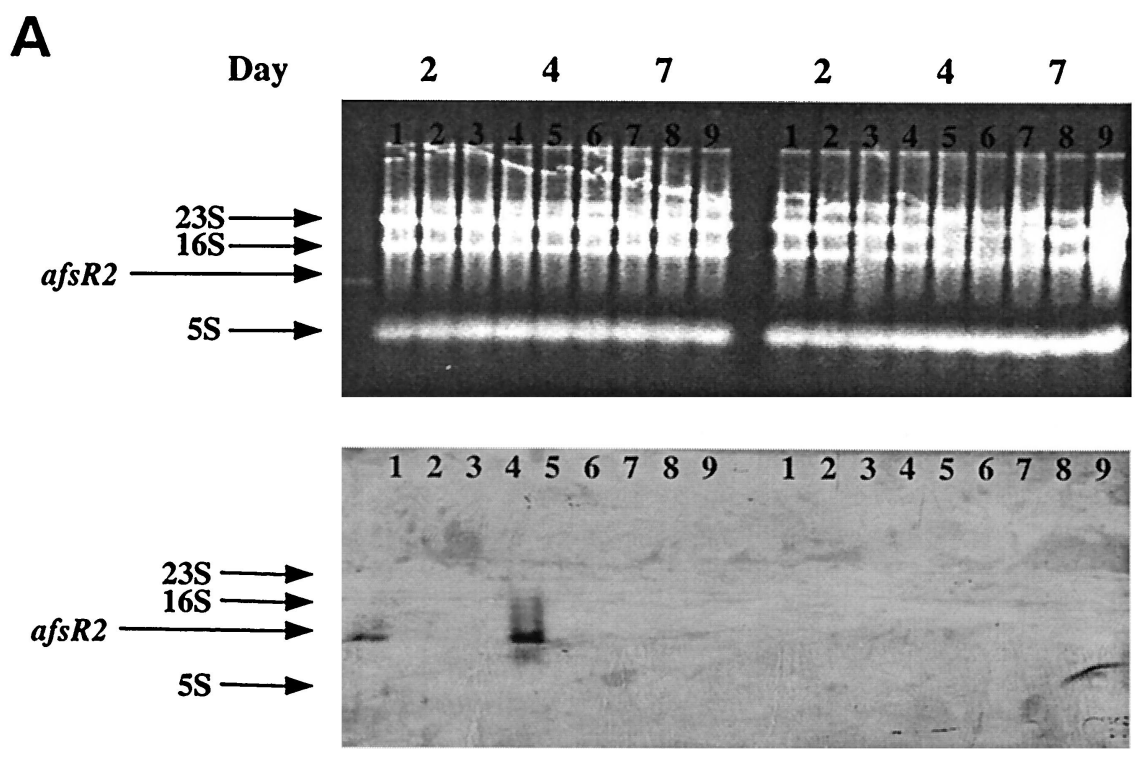

B $\begin{array}{lllllllllllllllllll}1 & 2 & 3 & 4 & 5 & 6 & 7 & 8 & 9 & 1 & 2 & 3 & 4 & 5 & 6 & 7 & 8 & 9\end{array}$

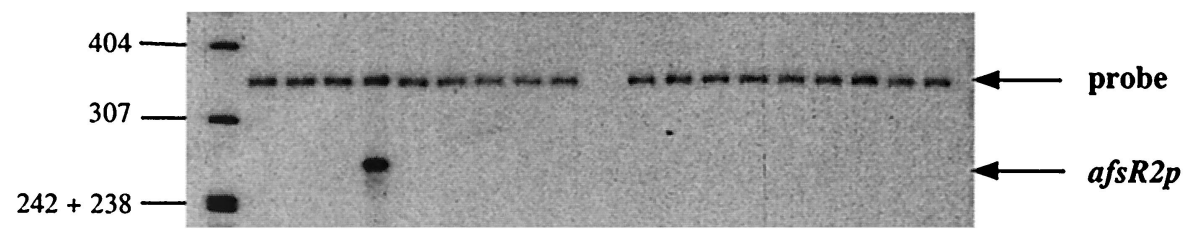

FIG. 4. (Top) Northern blot analysis of total RNA isolated from S. lividans TK21 (lanes 1, 4, and 7), SL94 (lanes 2, 5, and 8), and SL41 (lanes 3,6 , and 9) grown on minimal-medium plates containing either glycerol (left) or glucose (right) as the sole carbon source. Total RNAs from the three strains were isolated at day 2 (substrate mycelia; lanes 1, 2, and 3), day 4 (aerial mycelia; lanes 4, 5, and 6), and day 7 (spores; lanes 7, 8 , and 9). The top panel, which indicates equal loading of samples into lanes, shows the ethidium bromide-stained gel, indicating the locations of rRNA. The location of purified $a f s R 2$ mRNA isolated by prehybridization with afsR2 probe DNA which was the same as that used for $\mathrm{S} 1 \mathrm{mapping}$ (lane at extreme left). The lower panel shows the Northern blot. (Bottom) S1 nuclease protection analysis of transcription of afsR2 during development occurring in surface-grown cultures of S. lividans TK21 (lanes 1, 4, and 7), SL94 (lanes 2, 5, and 8), and SL41 (lanes 3, 6, and 9). RNA was isolated from cells grown on cellophane-covered minimal-medium plates containing either glycerol (left) or glucose (right) as the sole carbon source for 2 days (substrate mycelia; lanes 1,2, and 3), 4 days (aerial mycelia; lanes 4, 5, and 6), and 7 days (spores; lanes 7, 8, and 9). The uniquely $5^{\prime}$-end-labeled probes were prepared as described in Materials and Methods. A protected 282-bp fragment representing $a f_{s} R 2$ transcripts detected only in S. lividans TK21 $\left(a f_{s} R 2^{+}\right)$grown on glycerol minimal-medium plates for 4 days (lane 4$)$. The position of DNA size marker bands is shown to the left of the figure.

morphologically and physiologically, the two processes are independent (3). Streptomyces species commonly do not complete their morphological development or sporulate in liquid cultures but nevertheless synthesize the same antibiotics and other secondary metabolites that they produce on solid media during the formation of aerial mycelia and spores (3). Several pleiotropic loci that govern antibiotic production have been identified; some of these affect only antibiotic production, whereas others affect both antibiotic production and morphological differentiation, suggesting that the two processes share elements of genetic control $(3,4)$. To learn whether the life cycle-associated increased afsR 2 mRNA expression observed in cells cultured on solid media requires morphological differentiation per se, we examined the effect of carbon source on ACT production in cells grown in liquid. As shown in Fig. 5, strains TK21, SL94, and SL41 cultured in liquid minimal media (11) containing glucose as the sole carbon source all failed to produce ACT, as had been observed for solid medium. When glycerol was substituted for glucose as the carbon source, strain TK21, which contains functional $a f s R$ and afs $R 2$ genes, produced copious amounts of ACT, as had also occurred during growth on solid medium lacking glucose. Surprisingly however, we observed that, unlike plate-grown cells, the S. lividans strains utilizing glycerol as the sole carbon source also produced ACT efficiently in liquid medium when they carried deletions in afsR or afsR2, indicating that ACT biosynthesis had occurred by a pathway that is independent of both of these genes. Consistent with this conclusion was our finding that the growth of TK21 cells in glycerol-containing liquid media showed ACT synthesis in the absence of detectable afsR2 mRNA (Fig. 6).

Additional evidence that afsR2 mRNA production does not govern ACT production in liquid media was provided by the observation that cells grown in liquid media containing glucose as the sole carbon source failed to produce ACT, despite their ability to synthesize normal-size afsR2 transcripts (Fig. 6). 


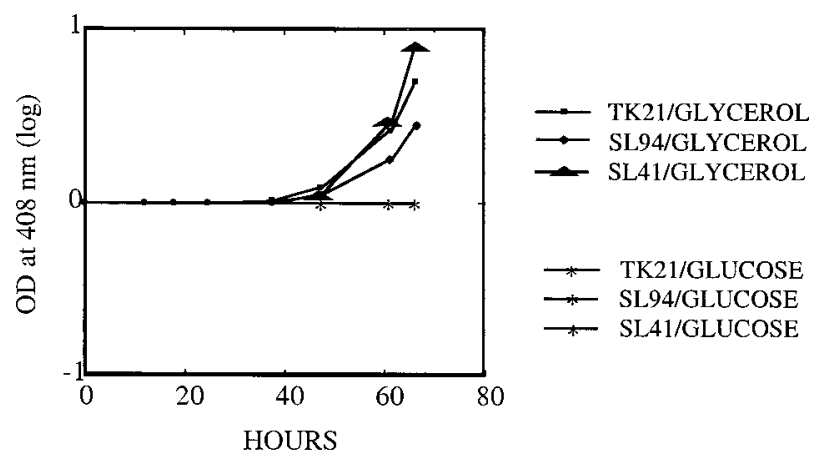

FIG. 5. Actinorhodin production by S. lividans (TK21, SL94, and SL41) in liquid culture. Each S. lividans was inoculated into a 250-ml baffled flask containing $25 \mathrm{ml}$ of minimal medium that included either glucose or glycerol as a sold carbon source, followed by incubation at $30^{\circ} \mathrm{C}$ for 3 days with shaking. Samples $(0.5 \mathrm{ml})$ were taken at various time points and measured.

Thus, under the liquid medium conditions we employed, regulation of the carbon source-dependent growth we observed for ACT biosynthesis in S. lividans may occur at a point downstream of the action of afs 2 or by an independent pathway. Interestingly, however, cells grown in liquid media containing glycerol produced high-molecular-weight transcripts detected by the afsR2 probe (Fig. 6, lane 1), raising the alternative possibility that afsR 2 mRNA in such cells may be initiated or terminated at a different location.

\section{DISCUSSION}

S. lividans has been widely studied for more than 20 years and has been used extensively as a host for DNA cloning in studies of Streptomyces biology. While S. lividans contains the complete biosynthetic pathway for ACT biosynthesis, this Streptomyces species has long been thought to be incapable of synthesizing significant amounts of ACT unless stimulated to do so by the introduction of multiple copies of regulatory genes such as afs $R$ and afsR2. The data presented here show that in $S$. lividans the choice of the carbon source is crucial to afs $R 2$ expression and consequently to ACT biosynthesis when only a single copy of $a f s R 2$ is present. During a search for conditions that upregulate expression of a single chromosomal copy

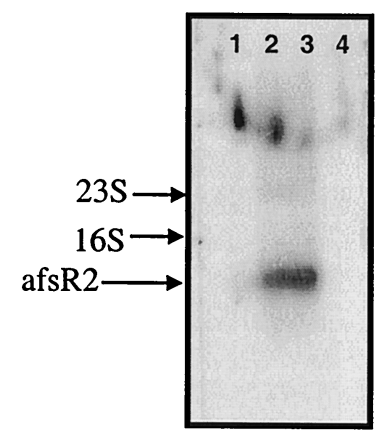

FIG. 6. Northern blot analysis of total RNA isolated from S. lividans grown for 3 days $30^{\circ} \mathrm{C}$ in minimal liquid culture. Lane 1, TK21 cultured with glycerol; lane 2, TK21 cultured with glucose; lane 3, TK21 cultured with both; lane 4, SL94 with glycerol.
afsR2, we found that ACT biosynthesis is subject to glucose repression during growth on solid media and that the use of glycerol as the sole carbon source enables S. lividans to produce the copious amount of deep-blue ACT pigment that has given $S$. coelicolor its species name $(19,22,25)$. This carbon source-dependent activation of ACT production is mediated through induction of mRNA synthesis initiated at the afs $R 2$ promoter, and it requires afs $R$. As derepression of the afs $R 2$ promoter during normal growth on solid media coincides temporally with the onset of the production of both aerial mycelia and ACT (Fig. 4), the turning on of ACT biosynthesis during morphological differentiation may occur at least in part by regulation at the level of afsR2 mRNA production.

Our studies also indicate the existence of a still-undefined separate carbon source-regulated ACT biosynthetic pathway that operates in S. lividans during growth in liquid media and is independent of both $a f s R$ and afsR2 (see also reference 3 ). Interestingly, the afsR2-dependent and afsR2-independent mechanisms of carbon source regulation of ACT synthesis in $S$. lividans have the same ultimate effect on ACT production despite their different effects on afs 22 expression. Whereas the $a f_{s} R$ and $a f_{s} R 2$ genes individually are not essential for the synthesis of ACT, multiple plasmid-borne copies of either the afs $R$ or the afsR2 gene can stimulate ACT production in $S$. coelicolor and S. lividans species independently of the carbon source. Potentially, this stimulation may occur by a direct gene dosage effect on protein production or by a mechanism in which the copies of $a f s R 2$ exceed the capacity of a repressor of $a f s S$. Whether the effect of a high afsR2 copy number is direct or occurs by activation of another regulator is not known.

Unlike S. lividans, S. coelicolor normally can produce large amounts of ACT during growth in glucose (7), suggesting either that AfsR2 synthesis in S. coelicolor is not sensitive to glucose repression or, alternatively, that ACT production on solid medium by this species does not depend on the activation of afsS (the $S$. coelicolor homolog of afsR2 [19]). Nevertheless, afs $R 2$ production in $S$. coelicolor, like afs $R 2$ production in $S$. lividans, requires expression of afsR (7).

Earlier experiments have shown the effects of carbon, source, nitrogen, phosphate, and other culture medium variables on antibiotic production in Streptomycetes $(1,2,6,17)$, and glucose repression of a variety of streptomycete promoters is known to occur $(15,16,20,23)$. The investigations reported here provide specific evidence for the physiological control of S. lividans regulatory genes that affect the expression of ACT. Accordingly, they raise the prospect that antibiotics apparently not synthesized by particular species under commonly used laboratory growth conditions may be under control of antibiotic biosynthetic regulatory genes that are subject to carbon source repression.

\section{ACKNOWLEDGMENTS}

This study was supported by NIH grant AI08619 to S.N.C. E.-S.K. was the recipient of a Dean's Postdoctoral Fellowship from the Stanford University School of Medicine.

E.-S.K. and H.-J.H. contributed equally to this work.

\section{REFERENCES}

1. Abou-Zeid, A. A., and A. A. Gammal. 1970. Effect of different carbon sources on erythromycin production by Streptomyces erythreus. Indian J. Exp. Biol. 8:146-147.

2. Aharonowitz, Y., and A. L. Demain. 1979. Nitrogen nutrition and regulation 
of cephalosporin production in Streptomyces clavuligerus. Can. J. Microbiol. 25:61-67.

3. Champness, W. C., and K. F. Chater. 1994. Regulation and integration of antibiotic production and morphological differentiation in Streptomyces, p. 61-93. In P. Piggot et al. (ed.), Regulation of bacterial differentiation. American Society for Microbiology, Washington, D.C.

4. Champness, W. C., P. Riggle, T. Adamidis, B. Kenney, and D. Aceti. 1993. Genetic elements involved in global antibiotic regulation in Streptomyces coelicolor, p. 227-233. In R. Baltz et al. (ed.), Industrial microorganisms: basic and applied molecular genetics. American Society for Microbiology, Washington D.C.

5. Chater, K. F. 1990. Multilevel regulation of Streptomyces differentiation. Trends Genet. 5:372-77.

6. Demain, A. L. 1979. Aminoglycosides, genes and regulation. Jpn. J. Antibiot. 32(Suppl): S15-S20.

7. Floriano, B., and M. J. Bibb. 1996. afsR is a pleiotropic but conditionally required regulatory gene for antibiotic production in Streptomyces coelicolor A3(2). Mol. Microbiol. 21:385-396.

8. Hara, O., S. Horinouchi, T. Uozumi, and T. Beppu. 1983. Genetic analysis of A-factor synthesis in Streptomyces coelicolor A3(2) and Streptomyces griseus. J. Gen. Microbiol. 129:2939-2944.

9. Hong, S. K., M. Kito, T. Beppu, and S. Horinouchi. 1991. Phosphorylation of the $A f s R$ product, a global regulatory protein for secondary-metabolite formation in Streptomyces coelicolor A3(2). J. Bacteriol. 173:2311-2318.

10. Hopwood, D. A. 1987. Towards an understanding of gene switching in Streptomyces: the basis of sporulation and antibiotic production. Proc. R. Soc. Lond. Series B. 235:2257-2269.

11. Hopwood, D. A., M. J. Bibb, K. J. Chater, T. Kieser, C. J. Bruton, H. M. Kieser, D. J. Lydiate, W. P. Smith, J. M. Ward, and H. Schrempf. 1985. Genetic manipulation of Streptomyces: a laboratory manual. The John Innes Foundation, Norwich, England.

12. Horinouchi, S., O. Hara, and T. Beppu. 1983. Cloning of a pleiotropic gene that positively controls biosynthesis of A-factor, actinorhodin, and prodigiosin in Streptomyces coelicolor A3(2) and Streptomyces lividans. J. Bacteriol. 155:1238-1248.

13. Horinouchi, S., M. Kito, M. Nishiyama, K. Furuya, S. K. Hong, K. Miyake, and T. Beppu. 1992. Primary structure of AfsR, a global regulatory protein for secondary metabolite formation in Streptomyces coelicolor A3(2). Gene 95:49-56.
14. Horinouchi, S., F. Malpartida, D. A. Hopwood, and T. Beppu. 1989. afs $B$ stimulates transcription of the actinorhodin biosynthetic pathway in Streptomyces coelicolor A3(2) and Streptomyces lividans. Mol. Gen. Genet. 215:355357.

15. Ingram, C., I. Delic, and J. Westpheling. 1995. ccrA1: a mutation in Streptomyces coelicolor that affects the control of catabolite repression. J. Bacteriol. 177:3579-3586.

16. Jones, G. H. 1986. Regulation of actinomycin synthesis in Streptomyces antibioticus. J. Nat. Prod. 49:981-987.

17. Lubbe, C., S. Wolfe, and A. L. Demain. 1985. Repression and inhibition of cephalosporin synthetases in Streptomyces clavuligerus by inorganic phosphate. Arch. Microbiol. 140:317-320.

18. Matsumoto, A., S. K. Hong, H. Ishizuka, S. Horinouchi, and T. Beppu. 1994. Phosphorylation of the AfsR protein involved in secondary metabolism in Streptomyces species by an eukaryotic-type protein kinase. Gene 146:47-56.

19. Matsumoto, A., H. Ishizukz, T. Beppu, and S. Horinouchi. 1995. Involvement of a small ORF downstream of the afs $R$ gene in the regulation of secondary metabolism in Streptomyces coelicolor A3(2). Actinomycetologica 9:37-43.

20. Ni, X., and J. Westpheling. 1997. Direct repeat sequences in the Streptomyces chitinase-63 promoter direct both glucose repression and chitin induction. Proc. Natl. Acad. Sci. USA 94:13116-13121.

21. Sambrook, J., E. F. Fritsch, and T. Maniatis. 1989. Molecular cloning: a laboratory manual, 2nd ed. Cold Spring Harbor Laboratory, Cold Spring Harbor, N.Y.

22. Stein, D., and S. N. Cohen. 1989. A cloned regulatory gene of Streptomyces lividans can suppress the pigment deficiency phenotype of different development mutants. J. Bacteriol. 171:2258-2261.

23. Steinmetz, M. 1993. Carbohydrate catabolism: pathways, enzymes, genetic regulation, and evolution, p. 157-70. In A. L. Sonenshein, J. A. Hoch, and R. Losick (ed.), Bacillus subtilis and other gram-positive bacteria: biochemistry, physiology, and molecular genetics. American Society for Microbiology, Washington, D.C.

24. Strauch, E., E. Takano, H. A. Baylis, and M. J. Bibb. 1991. The stringent response in Streptomyces coelicolor A3(2). Mol. Microbiol. 5:289-298.

25. Vogtli, M., P. C. Chang, and S. N. Cohen. 1994. afsR2: a previously undeleted gene encoding a 63-amino-acid protein that stimulates antibiotic production in Streptomyces lividans. Mol. Microbiol. 14:643-653. 